\title{
On integrability of Weingarten surfaces: a forgotten class $\ddagger$
}

\author{
Hynek Baran and Michal Marvan \\ Mathematical Institute in Opava, Silesian University in Opava, Na Rybníčku 1, 74601 Opava, \\ Czech Republic. E-mail: Michal.Marvan@math.slu.cz
}

\begin{abstract}
Rediscovered by a systematic search, a forgotten class of integrable surfaces is shown to disprove the Finkel-Wu conjecture. The associated integrable nonlinear partial differential equation

$$
z_{y y}+(1 / z)_{x x}+2=0
$$

possesses a zero curvature representation, a third-order symmetry, and a nonlocal transformation to the sine-Gordon equation $\phi_{\xi \eta}=\sin \phi$. We leave open the problem of finding a Bäcklund autotransformation and a recursion operator that would produce a local hierarchy.
\end{abstract}

AMS classification scheme numbers: 53A05, 35Q53

\section{Introduction}

With this paper, we launch a project to classify integrable classes of surfaces. These are classes of surfaces whose Gauss-Mainardi-Codazzi equations are integrable in the sense of soliton theory. Our long-term goals include obtaining lists of integrable classes as complete as computing resources permit, clarifying their mutual relations, and identifying known subcases. Our immediate goal is to demonstrate that the task is feasible and worth doing.

The classical geometry of immersed surfaces in the Euclidean space is well known to be closely connected with the modern theory of integrable systems [35]. The GaussWeingarten equations of a moving frame $\Psi$ always take the form

$$
\Psi_{x}=A \Psi, \quad \Psi_{y}=B \Psi .
$$

where $A, B$ are appropriate matrix functions. Integrability conditions of (1) are called the Gauss-Mainardi-Codazzi equations and take the form of a zero curvature representation

$$
A_{y}-B_{x}+[A, B]=0 .
$$

‡ This is an author-created, un-copyedited version of an article accepted for publication in J. Phys. A: Math. Theor. 42 (2009) 404007. IOP Publishing Ltd is not responsible for any errors or omissions in this version of the manuscript or any version derived from it. The definitive publisher authenticated version is available online at doi:10.1088/1751-8113/42/40/404007. 
Equation (2) is invariant under a huge group of gauge transformations

$$
A^{\prime}=S_{x} S^{-1}+S A S^{-1}, \quad B^{\prime}=S_{y} S^{-1}+S B S^{-1},
$$

induced by linear transformations $\Psi^{\prime}=S \Psi$ of the frame. Here $S$ is an invertible functional matrix, which can be restricted to take values in the Lie group $G$ associated with the Lie algebra $\mathfrak{g}$ matrices $A, B$ belong to - typically $\mathfrak{s o}(3)$.

The zero curvature representation (2) is the key ingredient in the soliton theory [15], where matrices $A, B$ are additionally assumed to depend on what is called the spectral parameter. The essential requirement for solitonic integrability is that the spectral parameter cannot be removed by means of the gauge transformation (3). Consequently, if the matrices $A, B$ can be modified so that they depend on a nonremovable parameter and still satisfy (2), then the corresponding Gauss-Mainardi-Codazzi equations are considered to be integrable in the sense of soliton theory, and their solutions are known as integrable or soliton surfaces [40].

Solitonic integrability can appear only when surfaces are subject to a constraint (such as being pseudospherical etc.). For numerous classical and recent examples see, e.g., the references $[4,35,38]$ (or [16] in the projective setting). Workable tools to classify such constraints include all the general integrability criteria [31], which are, however, not immediately applicable to non-evolutionary systems [30]. Other methods take advantage of the already known non-parametric zero curvature representation (2), e.g., the method of extended symmetries by Cieśliński et al. [10, 11, 12].

In this paper we employ a recent method due to one of us [29]. Its essence can be summarized as follows: We attempt to extend the given non-parametric zero curvature representation (a seed) to a power series in terms of the spectral parameter. In the work [29], the relevant computable cohomological obstructions are identified. Two obstacles make this procedure not entirely algorithmic: The parameter-dependent zero curvature representation can exist only in an extension of the Lie algebra $\mathfrak{g}$ and its jet order (the order of derivatives) can exceed that of the seed. If no obstructions are found, various ways exist to incorporate the true nonremovable parameter.

\section{Weingarten surfaces}

To be of genuine interest in geometry, the determining constraint on integrable surfaces must be invariant with respect to coordinate changes. The general non-differential invariant constraint is a functional relation $f(p, q)=0$ between the principal curvatures $p, q$. Such a functional relation is characteristic of Weingarten surfaces, which have been a topic of continuous interest, especially in global differential geometry [20, 41, 38, 26] and computer graphics [8]. Well known to be integrable is the class of linear Weingarten surfaces $[13,35]$, characterized by a linear relation

$$
a k+b h+c=0, \quad a, b, c=\text { const }
$$

between the Gauss curvature $k=p q$ and the mean curvature $h=\frac{1}{2}(p+q)$ (not to be mixed with a linear relation between the principal curvatures [23, 26]). Other integrable 
classes of Weingarten surfaces that sporadically occur in the literature all have a differential defining relation (e.g., the Hazzidakis equation of the Bonnet surfaces $[4,5,7]$; a harmonicity condition of Schief's [37] generalized linear Weingarten surfaces) or the class is not determined by the functional relation $f(p, q)=0$ alone (e.g., [9]).

So far, nothing contradicts the conjecture of Finkel [17, Conjecture 3.4] and $\mathrm{Wu}[43]$ that the only functional relation $f(p, q)=0$ to determine an integrable class of Weingarten surfaces is the linear relation (4). Supporting arguments include Wu's [43] proof of non-existence of an $\mathfrak{s o}(3)$-valued zero-curvature representation depending only on $x$-derivatives. Finkel's [17] argument roots in an unsuccessful search for higherorder symmetries and a (disputable, see [30, §2]) conjecture that integrability implies the existence of a local higher-order symmetry (actually the infinite hierarchy can be nonlocal, see also [31, 1 1.4.4.2]).

Nevertheless, the main result of the present paper asserts that the simple relation

$$
\frac{1}{p}-\frac{1}{q}=\text { const }
$$

between the main curvatures $p, q$, determines an integrable class of Weingarten surfaces. The associated nonlinear partial differential equation (21) has a parameter-dependent zero curvature representation (22) (outside the class considered in [43]), a third-order symmetry (24) (missed in [17]), and a recursion operator (25).

Paradoxically enough, surfaces satisfying relation (5) were not unknown to nineteenth century geometers. In view of their knowledge, our integrability result is not an entirely unexpected one. In fact, Ribaucour [34] established that the corresponding focal surfaces (evolutes) have a constant Gaussian curvature $k<0$ (are pseudospherical). Conversely, surfaces satisfying equation (5) are involutes of pseudospherical surfaces. Moreover, the classical Bianchi transformation [2] is nothing but the induced correspondence between the two focal pseudospherical surfaces. Ribaucour's theorems are covered in Darboux [13] and early twentieth-century monographs, such as [3, 14, 18, 42]. Later they became obsolete and forgotten as the induced Bianchi relation between pseudospherical surfaces became superseded by the classical Bäcklund transformation (the history is nicely reviewed by Prus and Sym in [32, Sect. 4]).

The first examples of surfaces satisfying relation (5) also date to the nineteenth century. Lipschitz [25] derived a four-parametric family in terms of elliptic integrals. A particular subcase, the rotation surface of von Lilienthal [24], is the involute surface of the pseudosphere.

The left-hand side of Equation (5) is equal to the difference of the principal radii of curvature at a point. This geometric quantity has a definite physical meaning, being associated with the interval of Sturm [39], also known as the astigmatic interval or the amplitude of astigmatism or simply the astigmatism [19]. A mirror or a refracting surface satisfying relation (5) will feature a constant amplitude of astigmatism in the normal directions. In the sequel, surfaces satisfying condition (5) will be called surfaces of constant astigmatism. Accordingly, the equation (21) to determine the surfaces of constant astigmatism will be called the constant astigmatism equation. 


\section{Preliminaries}

We shall consider surfaces $\mathbf{r}(x, y)$ parametrized by curvature lines. As is well known, the fundamental forms can be written as

$$
\begin{aligned}
& \mathrm{I}=u^{2} \mathrm{~d} x^{2}+v^{2} \mathrm{~d} y^{2}, \\
& \mathrm{II}=u^{2} p \mathrm{~d} x^{2}+v^{2} q \mathrm{~d} y^{2},
\end{aligned}
$$

where $p, q$ are the principal curvatures. Coordinates $x, y$ are unique up to arbitrary changes $x=X(x), y=Y(y)$.

Let $\Psi=\left(\mathbf{e}_{1}, \mathbf{e}_{2}, \mathbf{n}\right)$ denote the orthonormal frame, given by $\mathbf{e}_{1}=\mathbf{r}_{x} / u, \mathbf{e}_{2}=\mathbf{r}_{y} / v$, $\mathbf{n}=\mathbf{e}_{1} \times \mathbf{e}_{2}$. The Gauss-Weingarten equations

$$
\Psi_{x}=\left(\begin{array}{rrr}
0 & -\frac{u_{y}}{v} & u p \\
\frac{u_{y}}{v} & 0 & 0 \\
-u p & 0 & 0
\end{array}\right) \Psi, \quad \Psi_{y}=\left(\begin{array}{rrc}
0 & \frac{v_{x}}{u} & 0 \\
-\frac{v_{x}}{u} & 0 & v q \\
0 & -v q & 0
\end{array}\right) \Psi .
$$

are easily established. Their integrability conditions are the Gauss equation

$$
u u_{y y}+v v_{x x}-\frac{v}{u} u_{x} v_{x}-\frac{u}{v} u_{y} v_{y}+u^{2} v^{2} p q=0
$$

and the Mainardi-Codazzi equations

$$
(p-q) u_{y}+u p_{y}=0, \quad(q-p) v_{x}+v q_{x}=0
$$

Consequently, the two $\mathfrak{s o}(3)$ matrices occurring in formulas (6) constitute a nonparametric zero curvature representation of the Gauss-Mainardi-Codazzi system (7), (8). Because of the isomorphism $\mathfrak{s o}(3, \mathbb{C}) \cong \mathfrak{s l}(2, \mathbb{C})$, the same zero curvature representation can be alternatively written in terms of $2 \times 2$ matrices

$$
A_{0}=\left(\begin{array}{cc}
\frac{\mathrm{i} u_{y}}{2 v} & -\frac{1}{2} u p \\
\frac{1}{2} u p & -\frac{\mathrm{i} u_{y}}{2 v}
\end{array}\right), \quad B_{0}=\left(\begin{array}{cc}
-\frac{\mathrm{i} v_{x}}{2 u} & -\frac{1}{2} \mathrm{i} q v \\
-\frac{1}{2} \mathrm{i} q v & \frac{\mathrm{i} v_{x}}{2 u}
\end{array}\right) .
$$

Let us impose a constraint $f(p, q)=0$. If nontrivial, it can be resolved with respect to one of the curvatures, say

$$
q=F(p)
$$

which we assume henceforth. Then the Gauss-Mainardi-Codazzi system reduces substantially $[8,17,43]$. In particular, the Mainardi-Codazzi equations (8) have a general solution

$$
u=\frac{u_{0}}{E}, \quad v=-v_{0} E^{\prime}, \quad q=p-\frac{E}{E^{\prime}},
$$


where $E=E(p)$ is an arbitrary nonconstant function, $E^{\prime}=d E / d p$, and and $u_{0}, v_{0}$ are functions of $x$ and $y$, respectively, removable by transformation $\tilde{x}=\int u_{0} d x, \tilde{y}=\int v_{0} d y$. Therefore, we can put $u_{0}=-v_{0}=1$ without loss of generality, i.e.,

$$
u=\frac{1}{E}, \quad v=E^{\prime}, \quad q=p-\frac{E}{E^{\prime}} .
$$

The Gauss equation (7) then becomes

$$
p_{y y}=E^{3} E^{\prime \prime} p_{x x}+2 \frac{E^{\prime}}{E} p_{y}^{2}+E^{2}\left(E E^{\prime \prime}\right)^{\prime} p_{x}^{2}+E E^{\prime} p^{2}-E^{2} p .
$$

Summarizing, the Gauss-Mainardi-Codazzi system of Weingarten surfaces reduces to the single equation (12). The classification problem considered in this paper is "for which choices of the function $E(p)$ is the equation (12) integrable?"

By substituting (11) into (9), we easily obtain a nonparametric zero curvature representation of equation (12),

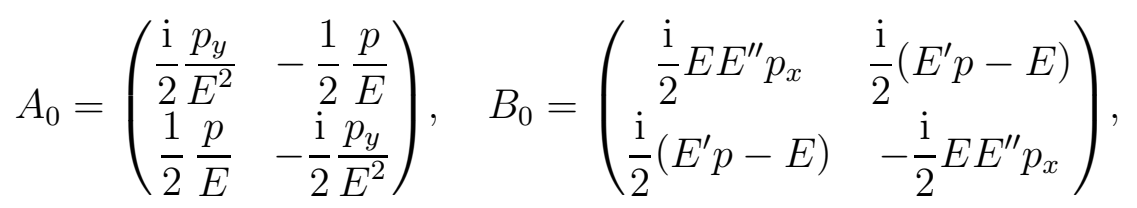

which will be the starting point of the calculations to follow.

\section{Cohomological criteria}

Readers not interested in details of the classification method can skip this section and continue to investigation of surfaces of constant astigmatism in Section 5.

We use the formal theory of partial differential equations, which treats coordinates, unknown functions, and their derivatives as independent quantities. Equations can be conveniently represented as submanifolds in appropriate jet spaces [6]. All our considerations being local, we let $J^{\infty}=J^{\infty}\left(\mathbb{R}^{2}, \mathbb{R}\right)$ denote the space of $\infty$-jets of smooth functions $\mathbb{R}^{2} \rightarrow \mathbb{R}$. The base $\mathbb{R}^{2}$ being equipped with coordinates $x, y$, the natural coordinates along fibres of $J^{\infty} \rightarrow \mathbb{R}^{2}$ correspond to $p$ and its derivatives. These will be denoted $p_{I}$, where $I$ stands for a symmetric multiindex in $x, y$ (including the "empty" multiindex $\emptyset$ such that $\left.p_{\emptyset}=p\right)$. The usual total derivatives

$$
D_{x}=\frac{\partial}{\partial x}+\sum_{I} p_{x I} \frac{\partial}{\partial p_{I}}, \quad D_{y}=\frac{\partial}{\partial y}+\sum_{I} p_{y I} \frac{\partial}{\partial p_{I}}
$$

can be viewed as acting on smooth functions defined on $J^{\infty}$ (by definition, a smooth function locally depends on a finite number of coordinates).

In $J^{\infty}$, we consider a submanifold $\mathcal{G}$ determined by equation (12) and all its differential consequences obtained by taking successive total derivatives of both sides of $(12)$. On $\mathcal{G}$, all derivatives of the form $p_{\text {Jyy }}$ become expressible in terms of the others. Therefore, derivatives $p_{I}$ with $y$ occurring no more than twice in $I$ serve as natural 
coordinates along the fibres of $\mathcal{G} \rightarrow \mathbb{R}^{2}$. Being tangent to (12), the total derivatives admit a restriction to $\mathcal{G}$. We retain the same notation $D_{x}, D_{y}$ for the restricted total derivatives.

The essence of the adopted point of view can be summarized as follows: A function $f$ on $J^{\infty}$ satisfies $\left.f\right|_{\mathcal{G}}=0$ if and only if $f$ is zero as a consequence of equation (12). From now on we assume that all objects (like the matrices $A, B$ ) are defined on $\mathcal{G}$. When writing

$$
\left.\left(D_{y} A-D_{x} B+[A, B]\right)\right|_{\mathcal{G}}=0
$$

we mean that the zero curvature condition (2) holds as a consequence of equation (12).

In what follows, characteristic elements $[27,28,36]$ play a crucial role. These are nonabelian analogues of characteristics of conservation laws [6]. For instance, the characteristic element of the initial zero curvature representation $(13)$ is the $\mathfrak{s l}(2, \mathbb{C})$ matrix

$$
C_{0}=\left(\begin{array}{cc}
\frac{\mathrm{i}}{2} \frac{1}{E^{2}} & 0 \\
0 & -\frac{\mathrm{i}}{2} \frac{1}{E^{2}}
\end{array}\right) .
$$

This immediately follows from the fact that

$$
D_{y} A_{0}-D_{x} B_{0}+\left[A_{0}, B_{0}\right]=C_{0} F
$$

where

$$
F=p_{y y}-E^{3} E^{\prime \prime} p_{x x}-2 \frac{E^{\prime}}{E} p_{y}^{2}-E^{2}\left(E E^{\prime \prime}\right)^{\prime} p_{x}^{2}-p^{2} E E^{\prime}-p E^{2}
$$

so that the Gauss equation (12) can be written as $F=0$.

Let $A=A(\lambda), B=B(\lambda)$ be the parametric zero curvature representation sought, $C=C(\lambda)$ the corresponding characteristic element. Besides (14), they will also satisfy the formula $[27]$

$$
\left.\sum_{I}(-\widehat{D})_{I}\left(\frac{\partial F}{\partial u_{I}^{k}} C\right)\right|_{\mathcal{G}}=0
$$

with $I$ running over all symmetric multiindices, including the empty one. Here $\widehat{D}_{x}=$ $D_{x}-[A, \cdot], \widehat{D}_{y}=D_{y}-[B, \cdot]$, the other values being obtained by composition, which can be taken in any order since (14) implies that $\widehat{D}_{x}, \widehat{D}_{y}$ commute.

Characteristic elements of gauge equivalent zero curvature representations are conjugate (similar). This allows us to transform characteristic elements into the normal form with respect to conjugation, namely, the Jordan normal form. Since the matrix $C_{0}$ above is diagonal, it follows that for $\lambda$ sufficiently close to zero the characteristic element $C(\lambda)$ will be also diagonalizable. 
However, diagonal matrices have a nontrivial stabilizer $\mathcal{S} \subset \mathrm{SL}(2, \mathbb{C})$ with respect to conjugation, which consists of diagonal matrices

$$
\left(\begin{array}{cc}
s & 0 \\
0 & 1 / s
\end{array}\right)
$$

Gauge transformations from the group $\mathcal{S}$ (henceforth $\mathcal{S}$-transformations) preserve the characteristic elements $C(\lambda)$. Their gauge action on a general $\mathfrak{s l}(2)$-valued zero curvature representation $A, B$ is sufficiently simple:

$$
\left(\begin{array}{cc}
a_{11} & a_{12} \\
a_{21} & -a_{11}
\end{array}\right) \mapsto\left(\begin{array}{cc}
\frac{s_{x}}{s}+a_{11} & s^{2} a_{12} \\
\frac{a_{21}}{s^{2}} & -\frac{s_{x}}{s}-a_{11}
\end{array}\right)
$$

and similarly for $B$. Using $\mathcal{S}$-transformations, one can achieve a unique normal form of matrices $A, B$ as follows: If $a_{12} \neq 0$, then by setting $s=\left(a_{21} / a_{12}\right)^{1 / 4}$ we turn $A$ into a symmetric matrix, while in the remaining case $a_{12}=0$ the zero curvature representation degenerates to a pair of conservation laws [28]. In other words, being symmetric is a normal form of nondegenerate zero curvature representations with respect to $\mathcal{S}$-transformations.

Turning back to our original problem, we see that $B_{0}$ is symmetric, and therefore the nearby matrices $B(\lambda)$ can also be symmetrized by an $\mathcal{S}$-transformation. A simple calculation shows that, by assuming diagonality of $C(\lambda)$ and symmetricity of $B(\lambda)$, we make the system (15) determined, hence solvable (actually, we fix the gauge).

Summarizing, the computation of zero curvature representation has been reduced to solution of the determined system (14), (15) under a suitable choice of normal forms for $C$ and $B$. However, this nonlinear system is still quite difficult to solve even with the help of computer algebra. To linearize the system, the work [29] considers Taylor expansions

$$
A(\lambda)=\sum_{k=0} A_{k} \lambda^{k}, \quad B(\lambda)=\sum_{k=0} B_{k} \lambda^{k}, \quad C(\lambda)=\sum_{k=0} C_{k} \lambda^{k},
$$

with $A_{0}, B_{0}, C_{0}$ coming from the initial parameterless zero curvature representation (9). The condition of zero curvature for $A(\lambda), B(\lambda)$ implies an infinite sequence of conditions of zero curvature for block triangular matrices

$$
A^{[m]}=\left(\begin{array}{cccc}
A_{0} & 0 & \cdots & 0 \\
A_{1} & A_{0} & \ddots & \vdots \\
\vdots & \ddots & \ddots & 0 \\
A_{m} & \cdots & A_{1} & A_{0}
\end{array}\right), \quad B^{[m]}=\left(\begin{array}{cccc}
B_{0} & 0 & \cdots & 0 \\
B_{1} & B_{0} & \ddots & \vdots \\
\vdots & \ddots & \ddots & 0 \\
B_{m} & \cdots & B_{1} & B_{0}
\end{array}\right) .
$$

Characteristic elements $C^{[m]}$ assume the same form. Zero curvature representations $A^{[m]}, B^{[m]}$ are to be considered under the gauge group consisting of block triangular 
matrices

$$
S^{[m]}=\left(\begin{array}{cccc}
E & 0 & \cdots & 0 \\
S_{1} & E & \ddots & \vdots \\
\vdots & \ddots & \ddots & 0 \\
S_{m} & \cdots & S_{1} & E
\end{array}\right) .
$$

with unit matrices $E$ in the diagonal positions. By a cohomological argument presented in [29, Prop. 1], a nontrivial family $A(\lambda), B(\lambda)$ with analytic dependence on $\lambda$ has expansions (16) such that $A_{1}$ or $B_{1}$ is not zero.

Let $(14)^{[m]},(15)^{[m]}$ denote the system obtained by substituting $A \rightarrow A^{[m]}, B \rightarrow B^{[m]}$ into system (14), (15), for arbitrary $m>0$. Observe that systems $(14)^{[m]},(15)^{[m]}$ are linear in their highest order unknowns $A_{m}, B_{m}, C_{m}$ and can be solved sequentially. Then the applicable cohomological criterion can be summarized as follows.

Proposition 1 ([29, Prop. 3]). Let $m>0$. If $A_{1}=B_{1}=0$ for all solutions $A^{[m]}, B^{[m]}$ of system $(14)^{[m]},(15)^{[m]}$, then there is no possibility to construct expansions (16) of order $m$, and consequently, the seed zero curvature representation $A_{0}, B_{0}$ cannot belong to a nontrivial analytic family.

Finally, to be able to solve system $(14)^{[m]},(15)^{[m]}$, we need to know the normal forms of matrices $A^{[m]}, B^{[m]}$. However, the normal forms for $B(\lambda), C(\lambda)$ established above immediately imply the same normal forms for $C_{k}$ (diagonal) and $B_{k}$ (symmetric).

\section{Results}

In this section, we present the results of computation of the cohomological obstructions in the case of the nonparametric zero curvature representation (13) of equation (12). As a sub-result we obtain the first few coefficients $A_{k}, B_{k}$ of Taylor expansions (16).

As we have seen in the preceding section (Proposition 1), the problem reduces to solving the system $(14)^{[\mathrm{m}]},(15)^{[\mathrm{m}]}$ of linear differential equations in total derivatives, for increasing values of $m$. This is only possible under a suitable restriction on the jet order of the unknowns $A_{k}, B_{k}, C_{k}, k>0$. To start with, we assume dependence on the first-order jets at most. Upon expanding all total derivatives, equations $(14)^{[\mathrm{m}]},(15)^{[\mathrm{m}]}$ become a large overdetermined system of linear partial differential equations. As such, the system is solvable by computing the passive (or involutive) form under a suitable (elimination) ranking [33].

Starting with $m=1$, we checked that nonzero matrices $A_{1}, B_{1}$ depending on second order derivatives exist for all possible determining relations (10). When incrementing $m$ to 2, nontrivial conditions started to appear, but we also reached the boundaries of our available computing resources. Consequently, our present classification results are still incomplete. Nevertheless, we were able to obtain a passive system of differential equations in several cases. Moreover, in two cases we were able to find $A_{2}, B_{2}$ explicitly. One of them were the linear Weingarten surfaces (4). Their integrability is a well 
established fact [35], the associated sine-Gordon equation $\phi_{x y}=\sin \phi$ being a textbook example of integrability. The other class emerged as a solution

$$
E=\frac{p}{\mathrm{e}^{1+c / p}}, \quad c=\text { const }
$$

of the ordinary differential equation

$$
\frac{E^{\prime \prime}}{E}-\left(\frac{E^{\prime}}{E}\right)^{2}+\frac{2}{p} \frac{E^{\prime}}{E}-\frac{1}{p^{2}}=0 .
$$

Henceforth we concentrate on the solution (18). The coefficients $u, v, q$ are easily found from (11) to be

$$
u=\frac{\mathrm{e}^{1+c / p}}{p}, \quad v=\frac{p+c}{p \mathrm{e}^{1+c / p}}, \quad q=\frac{p c}{p+c} .
$$

The last equality shows that the condition of constant astigmatism (5) holds with the constant $-1 / c$ on the right-hand side. The Gauss equation (12) becomes

$$
p_{y y}=\frac{c^{2}}{\mathrm{e}^{4\left(1+\frac{c}{p}\right)}} p_{x x}+2 \frac{p+c}{p^{2}} p_{y}^{2}+2 \frac{c^{2}(c-p)}{\mathrm{e}^{4\left(1+\frac{c}{p}\right)} p^{2}} p_{x}^{2}+\frac{c p^{2}}{\mathrm{e}^{2\left(1+\frac{c}{p}\right)}} .
$$

In principle, the cohomological method we applied can only prove nonintegrability and only indicate, but not prove, integrability. However, it was easy to guess an ansatz based on the form of $A_{k}$ and $B_{k}$. By solving (14), (15) we obtained a $\lambda$-dependent zero curvature representation

$$
\begin{aligned}
& A=\left(\begin{array}{cc}
\lambda c \frac{p_{x}}{p^{2}}+\sqrt{\lambda^{2}+\lambda} \mathrm{e}^{2\left(1+\frac{c}{p}\right) \frac{p_{y}}{p^{2}}} & \lambda \mathrm{e}^{1+2 \frac{c}{p}} \\
(\lambda+1) \mathrm{e} & -\lambda c \frac{p_{x}}{p^{2}}-\sqrt{\lambda^{2}+\lambda} \mathrm{e}^{2\left(1+\frac{c}{p}\right)} \frac{p_{y}}{p^{2}}
\end{array}\right), \\
& B=\left(\begin{array}{cc}
\lambda c \frac{p_{y}}{p^{2}}+\sqrt{\lambda^{2}+\lambda} c^{2} \mathrm{e}^{-2\left(1+\frac{c}{p}\right) \frac{p_{x}}{p^{2}}} & \sqrt{\lambda^{2}+\lambda} c \mathrm{e}^{-1} \\
\sqrt{\lambda^{2}+\lambda} c \mathrm{e}^{-1-2 \frac{c}{p}} & -\lambda c \frac{p_{y}}{p^{2}}-\sqrt{\lambda^{2}+\lambda} c^{2} \mathrm{e}^{-2\left(1+\frac{c}{p}\right)} \frac{p_{x}}{p^{2}}
\end{array}\right),
\end{aligned}
$$

which reduces to the initial $A_{0}, B_{0}$ given by (13) when $\lambda=-\frac{1}{2}$. The dependence on $p_{y}$ explains why this class of Weingarten surfaces is missing in Wu's paper [43].

Upon substitution

$$
x \rightarrow \frac{x}{|c|^{1 / 4}}, \quad y \rightarrow \frac{y}{|c|^{3 / 4}}, \quad p \rightarrow \frac{4 c}{2 \ln z+\ln |c|-4}
$$

the Gauss equation (12) simplifies to

$$
z_{y y}+\left(\frac{1}{z}\right)_{x x}+2=0
$$


and the zero-curvature representation (19) to

$$
\begin{aligned}
& A=\left(\begin{array}{cc}
\frac{1}{2} \sqrt{\lambda^{2}+\lambda} z_{y}+\frac{1+2 \lambda}{4} \frac{z_{x}}{z} & (\lambda+1) \sqrt{z} \\
\lambda \sqrt{z} & -\frac{1}{2} \sqrt{\lambda^{2}+\lambda} z_{y}-\frac{1+2 \lambda}{4} \frac{z_{x}}{z}
\end{array}\right), \\
& B=\left(\begin{array}{cc}
\frac{1}{2} \sqrt{\lambda^{2}+\lambda \frac{z_{x}}{z^{2}}+\frac{1+2 \lambda}{4} \frac{z_{y}}{z}} & \frac{\sqrt{\lambda^{2}+\lambda}}{\sqrt{z}} \\
\frac{\sqrt{\lambda^{2}+\lambda}}{\sqrt{z}} & -\frac{1}{2} \sqrt{\lambda^{2}+\lambda} \frac{z_{x}}{z^{2}}-\frac{1+2 \lambda}{4} \frac{z_{y}}{z}
\end{array}\right) .
\end{aligned}
$$

Let us remark that one can remove the $x$-derivatives from $A$ and $y$-derivatives from $B$ by the gauge transformation (3), albeit at the cost of introducing an exponential dependence on the spectral parameter. In (19) and (22), the corresponding gauge matrix is

$$
S=\left(\begin{array}{cc}
\mathrm{e}^{-\lambda c / p} & 0 \\
0 & \mathrm{e}^{\lambda c / p}
\end{array}\right) \text { and } S=\left(\begin{array}{cc}
z^{\lambda / 2} & 0 \\
0 & z^{-\lambda / 2}
\end{array}\right)
$$

respectively. For instance, the pair (22) becomes

$$
A^{\prime}=\left(\begin{array}{cc}
\frac{1}{2} \sqrt{\lambda^{2}+\lambda} z_{y} & (\lambda+1) z^{-\lambda} \\
\lambda z^{\lambda+1} & -\frac{1}{2} \sqrt{\lambda^{2}+\lambda} z_{y}
\end{array}\right), \quad B^{\prime}=\left(\begin{array}{cc}
\frac{1}{2} \sqrt{\lambda^{2}+\lambda} \frac{z_{x}}{z^{2}} & \sqrt{\lambda^{2}+\lambda} z^{-\lambda-1} \\
\sqrt{\lambda^{2}+\lambda} z^{\lambda} & -\frac{1}{2} \sqrt{\lambda^{2}+\lambda} \frac{z_{x}}{z^{2}}
\end{array}\right) .
$$

Equation (21) has obvious translational symmetries $\partial_{x}, \partial_{y}$, the scaling symmetry $2 z \partial_{z}-x \partial_{x}+y \partial_{y}$, and a discrete symmetry

$$
x \rightarrow y, \quad y \rightarrow x, \quad z \rightarrow \frac{1}{z}
$$

Computation reveals also two third-order symmetries of equation (21). One of them has the generator

$$
\begin{aligned}
& \frac{z^{3}}{K^{3}}\left(z_{x x x}-z z_{x x y}\right)-\frac{3}{K^{5}} z^{3}\left(z_{x}-z z_{y}\right)\left(z_{x x}-z z_{x y}\right)^{2} \\
& \quad-\frac{2}{K^{5}} z^{5}\left(9 z_{x}-z z_{y}\right) z_{x x}+\frac{1}{2 K^{5}} z^{2}\left(9 z_{x}^{2}+4 z z_{x} z_{y}-z^{2} z_{y}^{2}\right)\left(z_{x}-z z_{y}\right) z_{x x} \\
& \quad-\frac{2}{K^{5}} z^{3} z_{x}\left(z_{x}-z z_{y}\right)\left(4 z_{x}-z z_{y}\right) z_{x y}+\frac{4}{K^{5}} z^{6} z_{x} z_{x y} \\
& \quad+\frac{3}{K^{5}} z^{4}\left(5 z_{x}-z z_{y}\right) z_{x}^{2}-\frac{3}{K^{5}} z\left(z_{x}-z z_{y}\right) z_{x}^{4}
\end{aligned}
$$

where

$$
K=\sqrt{\left(z_{x}-z z_{y}\right)^{2}+4 z^{3}}
$$

The other is obtained by conjugation with the discrete symmetry (23). 
Moreover, A. Sergyeyev succeeded in finding a recursion operator for equation (21) in the usual pseudodifferential form

$$
-z_{y} D_{x}^{-1}+z_{x} D_{x}^{-2} D_{y}+2 z D_{x}^{-1} D_{y}
$$

(unpublished). As far as we could see, the operator generates only nonlocal symmetries. We leave as an open problem to find a recursion operator that would generate the third-order symmetry (24).

Let us conclude this section with some easy geometric observations. First of all, we can put $c=1$ without loss of generality. This can be always achieved by rescaling the ambient Euclidean metric and, if necessary, changing the orientation.

Now, the symmetries of the constant astigmatism equation (21) have the following geometric interpretation. Translation symmetries are simply reparametrizations of the surface. The scaling symmetry $\phi_{\varepsilon}: x \rightarrow \mathrm{e}^{\varepsilon} x, y \rightarrow \mathrm{e}^{-\varepsilon} y, z \rightarrow \mathrm{e}^{-2 \varepsilon} z$ takes a given surface $\mathbf{r}(x, y)$ to the parallel surface $\mathbf{r}(x, y)+\varepsilon \mathbf{n}(x, y)$. This is not surprising since parallel surfaces obviously have equal astigmatism in the corresponding points. Finally, swapping the orientation is another symmetry, which can be identified with a composition of the discrete symmetry (23) and the rescaling $\phi_{1}$. Hence, the discrete symmetry (23) corresponds to the change of the orientation followed by taking the parallel surface at the unit distance.

\section{Relation to pseudospherical surfaces}

As already mentioned in the introduction, nineteenth century geometers knew of a simple relation between pseudospheric surfaces and surfaces of constant astigmatism, even though they did not find the latter important enough to be named. In this section we reproduce some of their findings and derive a nonlocal transformation between the constant astigmatism equation (21) and the famous sine-Gordon equation. Again, we put $c=1$ for simplicity, meaning that the associated focal surfaces will be of Gaussian curvature -1 .

The forthcoming calculations are conveniently performed in terms of the variable $z$ given by formula $(20)$ or a new variable $w$ related to $z$ by

$$
z=\mathrm{e}^{2 w}
$$

Then we have

$$
u=(w-1) \mathrm{e}^{w}, \quad v=\frac{w}{\mathrm{e}^{w}}, \quad p=\frac{1}{w-1}, \quad q=\frac{1}{w} .
$$

and the discrete symmetry (23) becomes simply

$$
x \rightarrow y, \quad y \rightarrow x, \quad w \rightarrow-w .
$$

Given a surface $\mathcal{L}$, recall that its evolutes (also known as focal surfaces) are the loci of the principal centres of curvature of $\mathcal{L}$. Obviously, a generic surface $\mathcal{L}$ has two evolutes. They interchange positions under the change of the orientation. 
Proposition 2 (Ribaucour [34]). Evolutes of surfaces of constant astigmatism are pseudospherical surfaces.

Proof. Let $\mathbf{r}(x, y)$ be a surface parametrized by curvature lines. We use the orthonormal frame $\left(\mathbf{e}_{1}, \mathbf{e}_{2}, \mathbf{n}\right)$, where

$$
\mathbf{e}_{1}=\mathbf{r}_{x} / u, \quad \mathbf{e}_{2}=\mathbf{r}_{y} / v, \quad \mathbf{n}=\mathbf{e}_{1} \times \mathbf{e}_{2} .
$$

Then the two evolutes $\mathcal{L}^{\prime}$ and $\mathcal{L}^{\prime \prime}$ are given by

$$
\mathbf{r}^{\prime}=\mathbf{r}+\frac{\mathbf{n}}{p}, \quad \mathbf{r}^{\prime \prime}=\mathbf{r}+\frac{\mathbf{n}}{q}
$$

respectively. An easy calculation using the Gauss-Weingarten formulas (6) shows that

$$
\begin{aligned}
& \mathbf{r}_{x}^{\prime}=-\frac{p_{x}}{p^{2}} \mathbf{n}, \quad \mathbf{r}_{y}^{\prime}=-\frac{p_{y}}{p^{2}} \mathbf{n}+\left(1-\frac{q}{p}\right) \mathbf{r}_{y} \\
& \mathbf{r}_{x}^{\prime \prime}=-\frac{q_{x}}{q^{2}} \mathbf{n}+\left(1-\frac{p}{q}\right) \mathbf{r}_{x}, \quad \mathbf{r}_{y}^{\prime \prime}=-\frac{q_{y}}{q^{2}} \mathbf{n}
\end{aligned}
$$

the unit normals being

$$
\mathbf{n}^{\prime}=\frac{\mathbf{r}_{x}}{u}, \quad \mathbf{n}^{\prime \prime}=\frac{\mathbf{r}_{y}}{v} .
$$

Now assume $\mathbf{r}(x, y)$ to be a surface of constant astigmatism. By applying the substitutions (27) we obtain the first fundamental form of the evolutes in terms of $w$ :

$$
\begin{aligned}
& \mathrm{I}^{\prime}=\left(w_{x} d x+w_{y} d y\right)^{2}+\mathrm{e}^{-2 w} d y^{2}=d w^{2}+\mathrm{e}^{-2 w} d y^{2}, \\
& \mathrm{I}^{\prime \prime}=\mathrm{e}^{2 w} d x^{2}+\left(w_{x} d x+w_{y} d y\right)^{2}=\mathrm{e}^{2 w} d x^{2}+d w^{2}
\end{aligned}
$$

These are the well known pseudospherical metrics in terms of geodesic coordinates $w, y$ and $w, x$ on the first and the second sheet, respectively.

For further reference we also compute the second fundamental forms

$$
\mathrm{II}^{\prime}=-\mathrm{e}^{w} w_{x} d x^{2}+\frac{w_{x}}{\mathrm{e}^{3 w}} d y^{2}, \quad \mathrm{II}^{\prime \prime}=\mathrm{e}^{3 w} w_{y} d x^{2}-\frac{w_{y}}{\mathrm{e}^{w}} d y^{2} .
$$

Proposition 2 provides as with a couple of transformations from the constant astigmatism equation (21) to the sine-Gordon equation. To write them explicitly, we need to equip $\mathcal{L}^{\prime}$ and $\mathcal{L}^{\prime \prime}$ with the asymptotic coordinates $\xi, \eta$, i.e., the fundamental forms have to be

$$
\begin{aligned}
& \mathrm{I}^{\prime}=d \xi^{2}+2 \cos \phi^{\prime} d \xi d \eta+d \eta^{2}, \quad \mathrm{II}^{\prime}=2 \sin \phi^{\prime} d \xi d \eta, \\
& \mathrm{I}^{\prime \prime}=d \xi^{2}+2 \cos \phi^{\prime \prime} d \xi d \eta+d \eta^{2}, \quad \mathrm{II}^{\prime \prime}=2 \sin \phi^{\prime \prime} d \xi d \eta .
\end{aligned}
$$

Here $\phi^{\prime}$ and $\phi^{\prime \prime}$ are the angles between the coordinate lines on $\mathcal{L}^{\prime}$ and $\mathcal{L}^{\prime \prime}$, respectively. Using the previous expression of fundamental forms $\mathrm{I}^{\prime}, \mathrm{II}^{\prime}$ and $\mathrm{I}^{\prime \prime}, \mathrm{II}^{\prime \prime}$ in terms of the variable $w$, we easily see that $\xi, \eta$ can be obtained by the "reciprocal transformation" [35]

$$
\begin{aligned}
& d \xi=\frac{1}{2} \sqrt{\left(w_{x}+\mathrm{e}^{2 w} w_{y}\right)^{2}+\mathrm{e}^{2 w}} d x+\frac{1}{2} \sqrt{\left(\mathrm{e}^{-2 w} w_{x}+w_{y}\right)^{2}+\mathrm{e}^{-2 w}} d y, \\
& d \eta=\frac{1}{2} \sqrt{\left(w_{x}-\mathrm{e}^{2 w} w_{y}\right)^{2}+\mathrm{e}^{2 w}} d x-\frac{1}{2} \sqrt{\left(\mathrm{e}^{-2 w} w_{x}-w_{y}\right)^{2}+\mathrm{e}^{-2 w}} d y .
\end{aligned}
$$


These formulas are valid on both sheets and reflect another property established by Ribaucour [34], namely that asymptotic lines on $\mathcal{L}^{\prime}$ and $\mathcal{L}^{\prime \prime}$ correspond.

Then the angle $\phi^{\prime}$ associated with the first sheet satisfies

$$
\begin{aligned}
\cos \phi^{\prime} & =\frac{w_{x}^{2}-\mathrm{e}^{2 w}-\mathrm{e}^{4 w} w_{y}^{2}}{\sqrt{\left(w_{x}+\mathrm{e}^{2 w} w_{y}\right)^{2}+\mathrm{e}^{2 w}} \sqrt{\left(w_{x}-\mathrm{e}^{2 w} w_{y}\right)^{2}+\mathrm{e}^{2 w}}}, \\
\sin \phi^{\prime} & =-\frac{2 \mathrm{e}^{w} w_{x}}{\sqrt{\left(w_{x}+\mathrm{e}^{2 w} w_{y}\right)^{2}+\mathrm{e}^{2 w}} \sqrt{\left(w_{x}-\mathrm{e}^{2 w} w_{y}\right)^{2}+\mathrm{e}^{2 w}}},
\end{aligned}
$$

while the angle $\phi^{\prime \prime}$ associated with the second sheet satisfies a similar set of equations related by the substitution (28).

Proposition 3. Let $z(x, y)$ be a solution of the constant astigmatism equation (21), let $w=\frac{1}{2} \ln z$. Determine function $\phi^{\prime}$ by formula (30), and new coordinates $\xi, \eta$ by the reciprocal transformation (29). Then $\phi^{\prime}(\xi, \eta)$ is a solution of the sine-Gordon equation $\phi_{\xi \eta}=\sin \phi$.

Another solution of the sine-Gordon equation can be obtained by combination with the discrete symmetry (28). The other symmetries (translation and scaling) do not lead to essentially new solutions.

Now, it is easy to check that the evolutes of surfaces of constant astigmatism are related by the classical Bianchi transformation. Indeed, the corresponding points $\mathbf{r}^{\prime}$ and $\mathbf{r}^{\prime \prime}$ have a constant distance equal to $1 / p-1 / q$. The corresponding normals $\mathbf{n}^{\prime}=\mathbf{r}_{x} / u$ and $\mathbf{n}^{\prime \prime}=\mathbf{r}_{y} / v$ are orthogonal. Finally, being directed along the normal vector $\mathbf{n}$, the line joining the points $\mathbf{r}^{\prime}$ and $\mathbf{r}^{\prime \prime}$ is tangent to both surfaces $\mathcal{L}^{\prime}$ and $\mathcal{L}^{\prime \prime}$. These three properties characterize the classical Bianchi transformation. The Bianchi transformation is, however, superseded by the classical Bäcklund transformation [1], where the condition on the angle between the normals is relaxed from being right to being constant.

\section{Surfaces of constant astigmatism as involutes}

In principle, all surfaces of constant astigmatism can be obtained from solutions of the sine-Gordon equation as involute surfaces, see, e.g., Darboux [13, §802], Bianchi [3, $\S 130-\S 150]$ or Weatherburn [42, Ch. 8]. Geodesic nets on pseudospheric surfaces fall into three classes: hyperbolic, parabolic, and elliptic [3, §102]. Of them only the parabolic geodesic nets lead to surfaces of constant astigmatism $[3, \S 136]$.

Recall that the sine-Gordon $\phi_{\xi \eta}=\sin \phi$ describes surfaces of constant curvature -1 in the asymptotic coordinates $\xi, \eta$. By definition,

$$
\mathrm{I}=d \xi^{2}+2 \cos \phi d \xi d \eta+d \eta^{2}, \quad \mathrm{II}=2 \sin \phi d \xi d \eta,
$$

which leads to the Gauss-Weingarten equations

$$
\begin{array}{ll}
\mathbf{r}_{\xi \xi}=\frac{\cos \phi \mathbf{r}_{\xi}-\mathbf{r}_{\eta}}{\sin \phi} \phi_{\xi}, & \mathbf{r}_{\xi \eta}=\sin \phi \mathbf{n}, \quad \mathbf{r}_{\eta \eta}=\frac{\cos \phi \mathbf{r}_{\eta}-\mathbf{r}_{\xi}}{\sin \phi} \phi_{\eta} \\
\mathbf{n}_{\xi}=\frac{\cos \phi \mathbf{r}_{\xi}-\mathbf{r}_{\eta}}{\sin \phi}, & \mathbf{n}_{\eta}=\frac{\cos \phi \mathbf{r}_{\eta}-\mathbf{r}_{\xi}}{\sin \phi}
\end{array}
$$


Recall that coordinates $X, Y$ on a pseudospheric surface are called parabolic geodesic if the first fundamental form can be written as

$$
\mathrm{I}=d X^{2}+\mathrm{e}^{2 X} d Y^{2}
$$

To find the transformation from asymptotic to parabolic geodesic coordinates, observe that $d \xi^{2}+2 \cos \phi d \xi d \eta+d \eta^{2}=d X^{2}+\mathrm{e}^{2 X} d Y^{2}$ is equivalent to the system

$$
X_{\xi}^{2}+\mathrm{e}^{2 X} Y_{\xi}=1, \quad X_{\xi} X_{\eta}+\mathrm{e}^{2 X} Y_{\xi} Y_{\eta}=\cos \phi, \quad X_{\eta}^{2}+\mathrm{e}^{2 X} Y_{\eta}=1 .
$$

This system can be rewritten as

$$
\begin{array}{ll}
X_{\xi}=\cos \alpha, & Y_{\xi}=\mathrm{e}^{-X} \sin \alpha, \\
X_{\eta}=\cos \beta, & Y_{\eta}=\mathrm{e}^{-X} \sin \beta,
\end{array}
$$

and

$$
\phi=\alpha-\beta .
$$

In fact, (33) could be also $\phi=\beta-\alpha$, which can be reversed by changing the orientation of the surface. The new unknowns $\alpha$ and $\beta$ can be identified with the angles between the geodesics and the two asymptotic coordinate lines.

The integrability conditions of system (32) are

$$
\beta_{\xi}=-\sin \alpha, \quad \alpha_{\eta}=-\sin \beta,
$$

or, in view of relation (33),

$$
\beta_{\xi}=-\sin (\phi+\beta), \quad \beta_{\eta}=-\phi_{\eta}-\sin \beta .
$$

These are already compatible by virtue of the sine-Gordon equation. From equations (32) we obtain

$$
\mathbf{r}_{X}=-\frac{\sin \beta}{\sin \phi} \mathbf{r}_{\xi}+\frac{\sin \alpha}{\sin \phi} \mathbf{r}_{\eta}, \quad \mathbf{r}_{Y}=\left(\frac{\cos \beta}{\sin \phi} \mathbf{r}_{\xi}+\frac{\cos \alpha}{\sin \phi} \mathbf{r}_{\eta}\right) \mathrm{e}^{X}
$$

With respect to a given geodesic net, the involute surface $\tilde{\mathbf{r}}$ is composed of individual involute curves to the geodesics, based on one and the same orthogonal line $Y=$ const. Hence,

$$
\tilde{\mathbf{r}}=\mathbf{r}+(a-X) \mathbf{r}_{X},
$$

where $a$ is an arbitrary constant. With the help of equations (31), the fundamental forms $\tilde{\mathrm{I}}, \widetilde{\mathrm{II}}$ of the involute surface $\tilde{\mathbf{r}}$ can be routinely computed in asymptotic coordinates. In particular, the unit normal is $\tilde{\mathbf{n}}=\mathbf{r}_{X}$ and

$$
\begin{aligned}
\tilde{\mathrm{I}} & =\left(X^{2}-X+\frac{1}{2}\right)(1-\cos 2 \alpha) d \xi^{2}+(2 X-1)(\cos (\alpha+\beta)-\cos \phi) d \xi d \eta \\
& +\left(X^{2}-X+\frac{1}{2}\right)(1-\cos 2 \beta) d \eta^{2}, \\
\widetilde{\mathrm{II}} & =\left(X-\frac{1}{2}\right)(\cos 2 \alpha-1) d \xi^{2}+(\cos (\alpha+\beta)-\cos \phi) d \xi d \eta \\
& +\left(X-\frac{1}{2}\right)(\cos 2 \beta-1) d \eta^{2} .
\end{aligned}
$$


Hence, the principal radii of curvature are $X, X-1$. The Gauss-Mainardi-Codazzi equations of the involute surface hold as a consequence of the sine-Gordon equation, the two equations (32) on $X$ and the system (35) on $\beta$.

To obtain the corresponding solution of the constant astigmatism equation (21), we have to reparametrize the involute surfaces by curvature lines. Let $x, y$ denote the new coordinates. We choose $x=Y$ and define $y$ by the compatible system of equations

$$
y_{\xi}=\mathrm{e}^{X} \sin \alpha, \quad y_{\eta}=\mathrm{e}^{X} \sin \beta .
$$

A routine calculation shows that $\mathrm{e}^{-2 X(x, y)}$ is a solution of the constant astigmatism equation (21). Summarizing, we have the following proposition.

Proposition 4. Let $\phi(\xi, \eta)$ be a solution of the sine-Gordon equation $\phi_{\xi \eta}=\sin \phi$. Let $\alpha, \beta$ be solutions of the compatible equations

$$
\beta_{\xi}=-\sin \alpha, \quad \alpha_{\eta}=-\sin \beta, \quad \alpha-\beta=\phi .
$$

Determine functions $X, x, y$ by equations

$$
\begin{aligned}
& d X=\cos \alpha d \xi+\cos \beta d \eta \\
& d x=\mathrm{e}^{-X}(\sin \alpha d \xi+\sin \beta d \eta) \\
& d y=\mathrm{e}^{X}(\sin \alpha d \xi+\sin \beta d \eta)
\end{aligned}
$$

Then the function $\mathrm{e}^{-2 X(x, y)}$ is a solution of the constant astigmatism equation (21).

Example 1. Von Lilienthal's surfaces (involutes of the pseudosphere). Published in 1887, these surfaces seem to have fallen into oblivion. Recall that the pseudosphere is a surface obtained by rotating the tractrix around its asymptote. The meridians are geodesics of the parabolic type and therefore von Lilienthal's surface is obtained by rotating the involute of the tractrix (which itself is the involute of the catenary).

In geodesic coordinates $X, Y$, the "upper half" of the pseudosphere has a parametrization

$$
\mathbf{r}=\left(\begin{array}{c}
\mathrm{e}^{-X} \cos Y \\
\mathrm{e}^{-X} \sin Y \\
\operatorname{arcosh} \mathrm{e}^{X}-\sqrt{1-\mathrm{e}^{-2 X}}
\end{array}\right), \quad X>0,
$$

whose first fundamental form is $d X^{2}+\mathrm{e}^{-2 X} d Y^{2}$ (differs by the sign of $X$ from the canonical form used in the preceding section). Then

$$
\tilde{\mathbf{r}}=\mathbf{r}+(a-X) \mathbf{r}_{X}=\left(\begin{array}{c}
(X-a+1) \mathrm{e}^{-X} \cos Y \\
(X-a+1) \mathrm{e}^{-X} \sin Y \\
\operatorname{arcosh} \mathrm{e}^{X}-(X-a+1) \sqrt{1-\mathrm{e}^{-2 X}}
\end{array}\right), \quad X>0,
$$

parametrizes a rotational surface, for every real constant $a$. The surface is regular for all $a \leq 0$. Otherwise it has a cuspidal edge at $X=a$, which is a circle of radius $\mathrm{e}^{-a}$. 
Another singularity that occurs for every $a>1$ is the intersection with the rotation axis at $X=a-1$. Choosing the orientation so that the normal vector is

$$
\tilde{\mathbf{n}}=\left(\begin{array}{c}
-\mathrm{e}^{-X} \cos Y \\
-\mathrm{e}^{-X} \sin Y \\
\sqrt{1-\mathrm{e}^{-2 X}}
\end{array}\right)
$$

(i.e., $\mathbf{n}$ swaps orientation when crossing either of the singularities), then

$$
\begin{aligned}
& \tilde{\mathrm{I}}=\frac{(X-a)^{2}}{\mathrm{e}^{2 X}-1} d X^{2}+\frac{(X-a+1)^{2}}{\mathrm{e}^{2 X}} d Y^{2}, \\
& \widetilde{\mathrm{II}}=\frac{X-a}{\mathrm{e}^{2 X}-1} d X^{2}+\frac{X-a+1}{\mathrm{e}^{2 X}} d Y^{2} .
\end{aligned}
$$

and the principal radii of curvature are $X-a$ and $X-a+1$. The corresponding solution of the constant astigmatism equation $(21)$ is

$$
z=\frac{1}{x^{2}-\mathrm{e}^{2(a-1)}}
$$

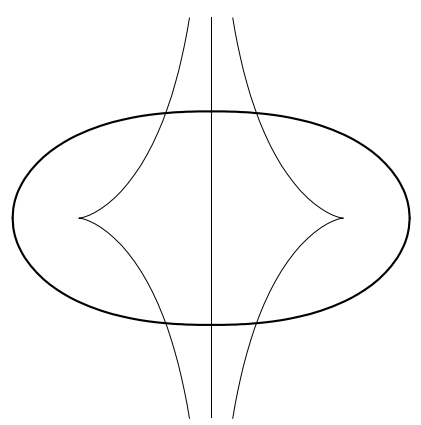

$a=-0.5$

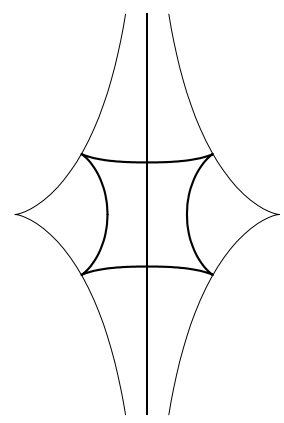

$a=0.7$

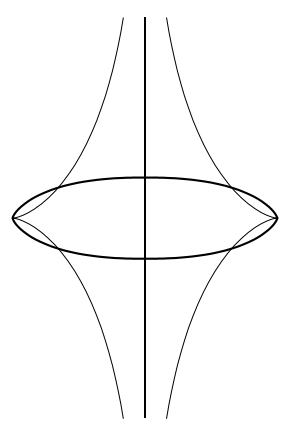

$a=0$

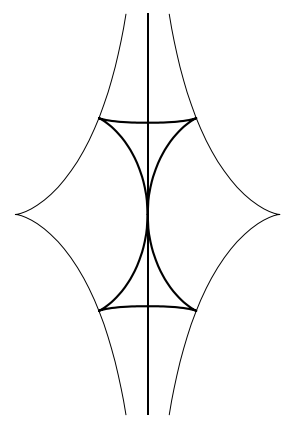

$a=1$

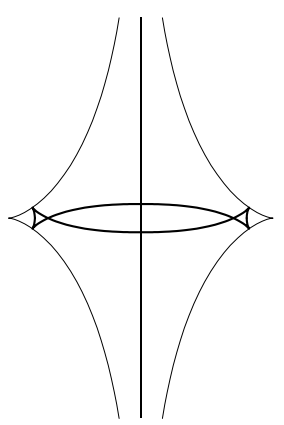

$a=0.2$

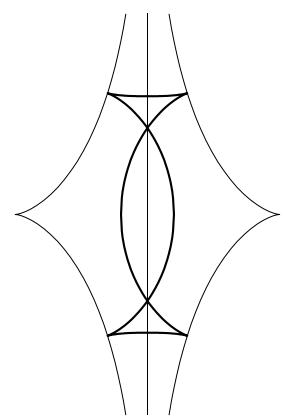

$a=1.2$

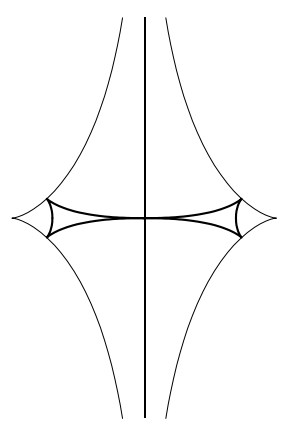

$a=1-\ln 2$

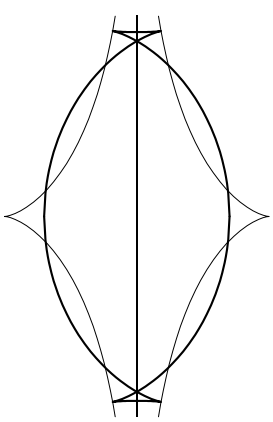

$a=1.7$

Figure 1. A gallery of von Lilienthal surfaces

Plane sections of von Lilienthal surfaces for various values of the parameter $a$ can be seen on Figure 1. Besides the rotation axis, each picture shows the tractrix, which is the plane section of the pseudosphere, and its involute curve, which is the plane section of the von Lilienthal surface. 
We finish this example with a short exploration of the behaviour at the limits of the definition domain. For $X=\infty$ the surface closes up at a point on the rotation axis at the height $a-1+\ln 2$, where both principal radii of curvature are infinite (the zero height is that of the cusp of the tractrix). For $X \rightarrow 0$ the surface vertically approaches a horizontal circle of diameter $|1-a|$. Two surfaces $\tilde{\mathbf{r}}(X, Y)$ and $-\tilde{\mathbf{r}}(X, Y)$ can be glued along this circle to form a single surface of constant astigmatism 1 . For $a=1$ both glued surfaces have a cusp here.

\section{Conclusions and discussion}

Among the still incomplete results of classification of integrable Weingarten surfaces, we have identified a class originally introduced and investigated by nineteenth-century geometers. The class, which we propose to call surfaces of constant astigmatism, is governed by the equation

$$
z_{y y}+\left(\frac{1}{z}\right)_{x x}+2=0
$$

For this equation we found an $\mathfrak{s l}(2)$-valued zero curvature representation depending on a parameter, a third-order symmetry, and a nonlocal transformation to the sineGordon equation $\phi_{\xi \eta}=\sin \phi$. We had to leave aside the problem of finding a Bäcklund transformation as well as a recursion operator producing a hierarchy of local symmetries.

It should be stressed that the classification problem of integrable surfaces is far from being easy. An obvious reason lies in the abundance of integrability-preserving ways to derive one surface from another. Clearly, parallel surfaces, evolutes, and involutes of integrable surfaces are integrable. On the differential equation level, the corresponding notion is that of the covering [22]. The integrable classes of surfaces must be either closed with respect to taking derived surfaces or the derivation must map one integrable class into another.

\section{Acknowledgments}

This paper would be impossible without encouragement, support and advice from J. Cieśliński, E. Ferapontov, R. López and A. Sergyeyev. The first-named author was supported by GAČR under project 201/07/P224. The second-named author by MŠMT under project MSM 4781305904. Thanks are also due to CESNET for granting access to the MetaCentrum computing facilities.

\section{References}

[1] A.V. Bäcklund, Om ytor med konstant negativ krökning, Lunds Univ. Arsskrift 19 (1883) 1-48.

[2] L. Bianchi, Ricerche sulle superficie a curvatura constante e sulle elicoidi, Tesi di Abilitazione, Ann. Scuola Norm. Sup. Pisa (I) 2 (1879) 285-304.

[3] L. Bianchi, Lezioni di Geometria Differenziale, Vol. I (E. Spoerri, Pisa, 1902). 
[4] A.I. Bobenko, Surfaces in terms of 2 by 2 matrices. Old and new integrable cases, in: A.P. Fordy and J.C. Wood, eds., Harmonic Maps and Integrable Systems, Aspects Math. E23 (Vieweg, Braunschweig, 1994), 83-127.

[5] A.I. Bobenko and U. Eitner, Bonnet surfaces and Painlevé equations, J. Reine Angew. Math. 499 (1998) 47-79.

[6] A.V. Bocharov, V.N. Chetverikov, S.V. Duzhin, N.G. Khor'kova, I.S. Krasil'shchik, A.V. Samokhin, Yu.N. Torkhov, A.M. Verbovetsky and A.M. Vinogradov, Symmetries and Conservation Laws for Differential Equations of Mathematical Physics, Transl. Math. Monographs 182 (Amer. Math. Soc., Providence, 1999).

[7] O. Bonnet, Mémoire sur la théorie des surfaces applicables sur une surface donnée, Journal de l'École Polytechnique 42 (1867) 1-155.

[8] B. van-Brunt, Weingarten surfaces, in: R.B. Fisher, ed., Design and Application of Curves and Surfaces, Mathematics of Surfaces V (Clarendon Press, Oxford, 1994) 49-87.

[9] Ö. Ceyhan, A. Fokas and M. Gürses, Deformations of surfaces associated with integrable GaussMainardi-Codazzi equations, J. Math. Phys. 41 (2000) 2251-2270.

[10] J. Cieśliński, Lie symmetries as a tool to isolate integrable symmetries, in: M. Boiti et al., eds., Nonlinear Evolution Equations and Dynamical Systems (World Scientific, Singapore, 1992).

[11] J. Cieśliński, Non-local symmetries and a working algorithm to isolate integrable symmetries, $J$. Phys. A: Math. Gen. 26 (1993) L267-L271.

[12] J. Cieśliński, P. Goldstein and A. Sym, On integrability of the inhomogeneous Heisenberg ferromagnet model: examination of a new test, J. Phys. A: Math. Gen. 27 (1994) 1645-1664.

[13] G. Darboux, Leçons sur la théorie générale des surface et les applications géométriques du calcul infinitésimal, Vol. III (Chelsea, Bronx, NY, 1972).

[14] L.P. Eisenhart A Treatise on the Differential Geometry of Curves and Surfaces (Ginn, Boston, 1909).

[15] L.D. Faddeev and L. Takhtajan, Hamiltonian Methods in the Theory of Solitons, Classics in Mathematics (Springer, Berlin, 1987).

[16] E.V. Ferapontov, Integrable systems in projective differential geometry, Kyushu J. Math. 54 (2000) $183-215$.

[17] F. Finkel, On the integrability of Weingarten surfaces, in: A. Coley et al., ed., Bäcklund and Darboux Transformations. The Geometry of Solitons, AARMS-CRM Workshop, June 4-9, 1999, Halifax, N.S., Canada, (Amer. Math. Soc., Providence, 2001) 199-205.

[18] A.R. Forsyth, Lectures on the Differential Geometry of Curves and Surfaces (Cambridge Univ. Press, Cambridge, 1920).

[19] H.J. Gray and A. Isaacs, eds., Dictionary of Physics, 3rd ed., Longman, London, 1991.

[20] H. Hopf, Über Flächen mit einer Relation zwischen den Hauptkrümmungen, Math. Nachr. 4 (1951) $232-249$

[21] J.G. Kingston and C. Rogers, Reciprocal Bäcklund transformations of conservation laws, Phys. Lett. A 92 (1982) 261-264.

[22] I.S. Krasil'shchik and A.M. Vinogradov, Nonlocal trends in the geometry of differential equations: symmetries, conservation laws, and Bäcklund transformations, Acta Appl. Math. 15 (1989) 161209.

[23] W. Kühnel and M. Steller, On closed Weingarten surfaces, Monatsh. Math. 146 (2005) 113-126.

[24] R. von Lilienthal, Bemerkung über diejenigen Flächen bei denen die Differenz der Hauptkrümmungsradien constant ist, Acta Mathematica 11 (1887) 391-394.

[25] R. Lipschitz, Zur Theorie der krummen Oberflächen, Acta Math. 10 (1887) 131-136.

[26] R. López, On linear Weingarten surfaces, Internat. J. Math. 19 (2008) 439-448.

[27] M. Marvan, On zero curvature representations of partial differential equations, in: Differential Geometry and Its Applications, Proc. Conf. Opava, Czechoslovakia, Aug. 24-28, 1992 (Silesian University, Opava, 1993) 103-122; http://www.emis.de/proceedings/5ICDGA.

[28] M. Marvan, A direct method to compute zero curvature representations. The case $s l_{2}$, in: Proc. 
Conf. Secondary Calculus and Cohomological Physics, Moscow, Russia, Aug. 24-31, 1997; http://www.emis.de/proceedings/SCCP97.

[29] M. Marvan, On the spectral parameter problem, Acta Appl. Math., to appear.

[30] A.V. Mikhailov, V.S. Novikov and J.P. Wang, On classification of integrable non-evolutionary equations, Stud. Appl. Math. 118 (2007) 419-457.

[31] A.V. Mikhailov and V.V. Sokolov, Symmetries of differential equations and the problem of integrability, in: A.V. Mikhailov, ed., Integrability, Lect. Notes Phys. 767 (Springer, 2008).

[32] R. Prus and A. Sym, Rectilinear congruences and Bäcklund transformations: roots of the soliton theory, in: D. Wójcik and J. Cieśliński, Nonlinearity $\&$ Geometry, Luigi Bianchi Days, Proc. 1st Non-Orthodox School, Warsaw, September 21-28, 1995 (Polish Scientific, Warsaw, 1998) 25-36.

[33] G.J. Reid, P. Lin and A.D. Wittkopf, Differential elimination-completion algorithms for DAE and PDAE, Stud. Appl. Math. 106 (2001) 1-45.

[34] A. Ribaucour, Note sur les développées des surfaces, C. R. Acad. Sci. Paris 74 (1872) 1399-1403.

[35] C. Rogers and W.K. Schief, Bäcklund and Darboux Transformations. Geometry and Modern Applications in Soliton Theory (Cambridge Univ. Press, Cambridge, 2002).

[36] S.Y. Sakovich, On zero-curvature representations of evolution equations, J. Phys. A: Math. Gen. 28 (1995) 2861-2869.

[37] W.K. Schief, On Laplace-Darboux-type sequences of generalized Weingarten surfaces, J. Math. Phys. 41 (2000) 6566-6599.

[38] W.K. Schief and B.G. Konopel'chenko, On the unification of classical and novel integrable surfaces, I. Differential geometry, Proc. Royal Soc. A 459 (2003) 67-84.

[39] C. Sturm, Mémoire sur la théorie de la vision, C.R. Acad. Sci. Paris 20 (1845) 554-560, 761-767, 1238-1257.

[40] A. Sym, Soliton surfaces and their applications. Soliton geometry from spectral problems, in: R. Martini, ed., Geometric Aspects of the Einstein Equations and Integrable Systems, Lecture Notes in Physics 239 (Springer, Berlin, 1985) 154-231.

[41] K. Voss, Über geschlossene Weingartensche Flächen, Math. Ann. 138 (1959) 42-54.

[42] C.E. Weatherburn, Differential Geometry of Three Dimensions (Cambridge University Press, Cambridge, 1927).

[43] Hongyou Wu, Weingarten surfaces and nonlinear partial differential equations, Ann. Global Anal. Geom. 11 (1993) 49-64. 